\title{
Hepatic perfusion changes in patients with liver metastases: comparison with those patients with cirrhosis
}

\author{
E Leen, J A Goldberg, J R Anderson, J Robertson, B Moule, T G Cooke, C S McArdle
}

\begin{abstract}
Previous studies using dynamic scintigraphy have shown that the measurement of changes in hepatic perfusion may be exploited to detect liver metastases. Similar hepatic haemodynamic changes also occur in cirrhosis, however, thereby reducing the diagnostic power of the technique. The ability of duplex colour Doppler sonography (DCDS) to differentiate between the changes in liver perfusion in patients with cirrhosis and those with hepatic metastases was assessed. Hepatic arterial and portal venous blood flows were measured in $\mathbf{3 0}$ control subjects, 20 patients with cirrhosis, and 55 patients with overt liver metastases. The Doppler perfusion index (DPI) (the rate of hepatic arterial to total liver blood flow) and the congestive index (ratio of the cross sectional area of the vessel to time averaged velocity of blood flow in the vessel) of the hepatic artery (HCI) and portal vein (PCI) were calculated. The hepatic arterial blood flow of the cirrhotic and metastatic groups was significantly raised compared with that of controls, and the portal venous blood flow of the former groups were reduced $(p<0.0001)$. The DPIs of the cirrhotic and metastatic groups were therefore significantly raised compared with those of controls $(p<0 \cdot 0001)$. No significant difference was noted in HCI values between the three groups. The PCI values of the cirrhotic group, however, were significantly raised compared with those of controls and patients with metastases $(\mathbf{p}<0.0001)$. The data suggest that DCDS measurement of PCI may be of value in differentiating between the hepatic perfusion changes caused by cirrhosis and those resulting from hepatic metastases, thereby increasing the diagnostic power of this technique.

(Gut 1993; 34: 554-557)
\end{abstract}

The normal liver receives about $25 \%$ of its blood supply via the hepatic artery and the remainder through the portal vein. Parkin et al postulated that the presence of liver tumour might increase the hepatic arterial blood flow, as the blood supply of the tumour is almost exclusively arterial.' using dynamic scintigraphy as an indirect method of measuring blood flow, they calculated the ratio of hepatic arterial to total liver blood flow, the hepatic perfusion index (HPI), and found that it was raised in patients with liver metastases. Leveson et al went on to suggest that HPI may be useful in the diagnosis of 'occult' colorectal liver metastases.' A well recognised deficiency of dynamic scintigraphy, however, is that HPI is also raised in the presence of hepatic cirrhosis thereby reducing the diagnostic power of the technique. ${ }^{3}$

In a previous study using duplex/colour Doppler sonography (DCDS), we also showed that hepatic metastases may be detected by measuring the subtle changes in hepatic perfusion. ${ }^{+5}$ We found that the Doppler perfusion index (DPI) was abnormally high in the presence of liver metastases. The effects of cirrhosis on the DPI have not been evaluated previously. In contrast to dynamic scintigraphy, DCDS is a more direct method of flow measurement, and the much greater information provided should allow the haemodynamic effects of benign and malignant liver disease to be clearly differentiated.

In this study, we investigated the flow changes associated with hepatic metastases and cirrhosis to assess the value of DCDS in differentiating between the hepatic haemodynamic changes in patients with cirrhosis and those with colorectal liver metastases.

\section{Methods}

Thirty healthy volunteers (age range 23-77 years), 55 patients with histologically proved overt colorectal liver metastases (age range 31-75 years), and 20 patients with cirrhosis (age range 37-70 years) were included in the study. The cirrhosis was caused by alcohol abuse and all these patients had oesophageal varices at endoscopy. The severity of cirrhosis was evaluated according to Child-Pugh's classification. The score of the patients ranged from 2 to 13 with a mean of $7 \cdot 1$.

All subjects were examined in the supine position after 12 hours of fasting. DCDS was used to calculate the hepatic arterial and portal venous blood flows from the product of the measured time average velocity of blood in the vessel and the time average cross sectional area of the lumen of the vessel. The total liver blood flow was taken as the sum of the hepatic arterial and the portal venous blood flow. The DPI, defined as the ratio of the hepatic arterial to total liver blood flow was calculated. The congestive indices of the hepatic artery (HCI) and portal vein (PCI) were calculated as the ratio of the time average cross sectional area of the vessel to the time average velocity of blood flow within the vessel. The ratio of the cross sectional area of the hepatic artery to the portal vein and the hepatic arterial resistive index (HARI) as described in the technical section were calculated. 
Haemodynamic results for control subjects and patients with hepatic metastases and those with cirrhosis

\begin{tabular}{llll}
\hline & $\begin{array}{l}\text { Controls } \\
(n=30) \\
(\text { mean }(S D))\end{array}$ & $\begin{array}{l}\text { Hepatic } \\
\text { metastases } \\
(n=55) \\
(\text { mean }(S D))\end{array}$ & $\begin{array}{l}\text { Cirrhotics } \\
(n=20) \\
(\text { mean }(S D))\end{array}$ \\
\hline HABF & $194(125)$ & $633(338)^{\star}$ & $453(248)^{\star}$ \\
PVBF & $1200(572)$ & $691(344)^{\star}$ & $604(507)^{\star}$ \\
DPI & $0 \cdot 14(0 \cdot 06)$ & $0 \cdot 49(0 \cdot 13)^{\star}$ & $0 \cdot 48(0 \cdot 19)^{\star}$ \\
HCI & $0 \cdot 011(0 \cdot 004)$ & $0 \cdot 012(0.013)$ & $0 \cdot 009(0 \cdot 004)$ \\
PCI & $0 \cdot 095(0.051)$ & $0 \cdot 102(0.055)$ & $0 \cdot 329(0 \cdot 303)^{\star}$ \\
HARI & $0 \cdot 79(0 \cdot 04)$ & $0 \cdot 70(0.07)^{\star}$ & $0 \cdot 72(0 \cdot 04)^{\star}$ \\
TLBF & $1394(642)$ & $1304(583)$ & $966(425) \dagger$ \\
AR & $0 \cdot 14(0.04)$ & $0 \cdot 34(0 \cdot 17)^{\star}$ & $0 \cdot 19(0 \cdot 12)$ \\
\hline
\end{tabular}

HABF: hepatic arterial blood flow $\left(\mathrm{cm}^{3} / \mathrm{min}\right)$; PVBF: portal venous blood flow $\left(\mathrm{cm}^{3} / \mathrm{min}\right)$; TLBF: total liver blood flow $\left(\mathrm{cm}^{3} /\right.$ min); AR: area ratio of hepatic artery to portal vein; HARI: hepatic arterial resistive index; HCI: hepatic (arterial) congestive index $(\mathrm{cms})$; PCI: portal congestive index $(\mathrm{cms})$; DPI. hepatic arterial to total blood flow. ${ }^{\star}: p<0.0001 ; t: p<0.0004$

TECHNIQUE OF DOPPLER FLOW MEASUREMENTS A Diasonics Spectra scanner (Diasonics Sonotron Ltd, Bedford, UK) which consists of duplex and colour Doppler facilities was used with a $3.5 \mathrm{MHz}$ convex, phased linear array probe. In the Doppler mode, ultrasound waves were emitted and received by a single $3.5 \mathrm{MHz}$ probe at a frequency of $3 \mathrm{MHz}$, with a repetition frequency of $3.7 \mathrm{kHz}$. It was possible to steer the Doppler beam to any location, and the angle between the axis of the Doppler beam to that of the vessel examined could be measured from the monitor. An angle range of $50-68^{\circ}$ was used for velocity measurement. Spectral analysis was performed using fast Fourier transformation and the Doppler shift signal was displayed on the monitor. The system was equipped with software to compute the time average velocity from the velocity spectral display automatically after placement of the calipers at the start and end of one or more cardiac cycles. The cross sectional area of the vessel was measured by mapping the perimeter of the vessel lumen with the 'tracker ball'.

A transverse scan was made at the epigastrium to locate the common hepatic artery in its longitudinal axis. The Doppler cursor was placed over the lumen of the artery segment as near to the origin as possible, at the point where it first became horizontally straight. The Doppler sample volume and beam angle were

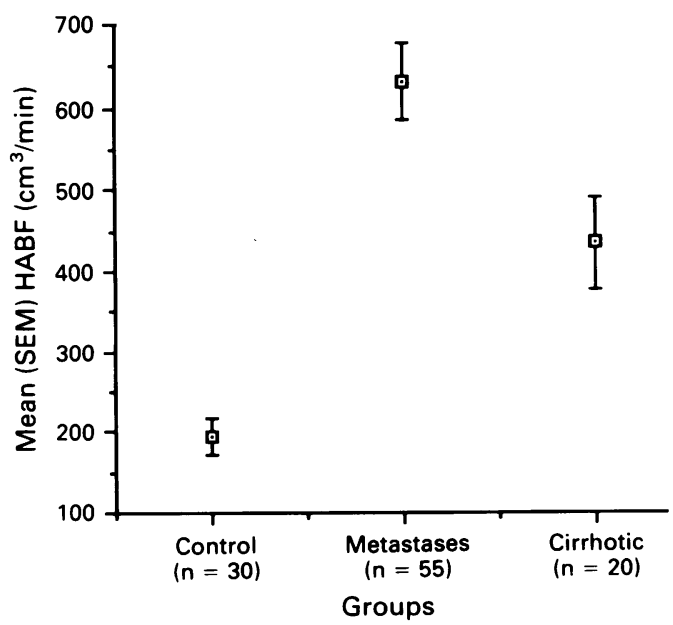

Figure 1: Hepatic arterial blood flow $(H A B F)$ in control subjects and in patients with hepatic metastases and those with cirrhosis. adjusted, and the time average velocity was calculated over four cardiac cycles. The cross sectional area of the artery was measured at the same point at right angles to the vessel. The time average cross sectional area was calculated by taking the mean of the areas measured separately over four different cardiac cycles. The same parameters were obtained for the portal vein in a similar manner at the mid level of the vein. All measurements were performed under respiratory suspension in expiration to allow optimal visualisation of the portal vein and enable a more acute angle to be achieved for Doppler purposes. Each measurement was performed repeatedly until satisfactory spectral patterns were obtained.

The resistive index of the hepatic artery (HARI) was measured automatically from the velocity spectral display using the onboard software, after placement of the calipers at the peak systolic velocity and at the end diastolic velocity.

Informed consent was obtained from all the patients. All the data were analysed using the Mann-Whitney $U$ test and the Kruskal-Wallis test.

\section{Results}

The results for controls, patients with cirrhosis, and for those with overt liver metastases are summarised in the Table. Compared with controls, patients in the cirrhotic and metastatic groups had a significant increase in hepatic arterial blood flow $(p<0.0001$ and $p<0.0001$ respectively, Fig 1 ) and also a significant reduction in portal venous blood flow $(\mathrm{p}<0.0001$ and $\mathrm{p}<0.0001$ respectively, Fig 2). These changes therefore resulted in an abnormal increase in the DPIs $(p<0.0001$ and $p<0.0001)$ compared with controls (Fig 3). No significant difference was noted in the total liver blood flow between the controls and the metastatic group. It was significantly reduced, however, in patients with cirrhosis $(\mathrm{p}<0.0004$ and $\mathrm{p}<0.0130$ respectively).

The increase in the PCIs of the cirrhotic groups was highly significant compared with that in both controls and patients with metastases $(p<0.0001$ and $p<0.0001$, respectively); (Fig 4).

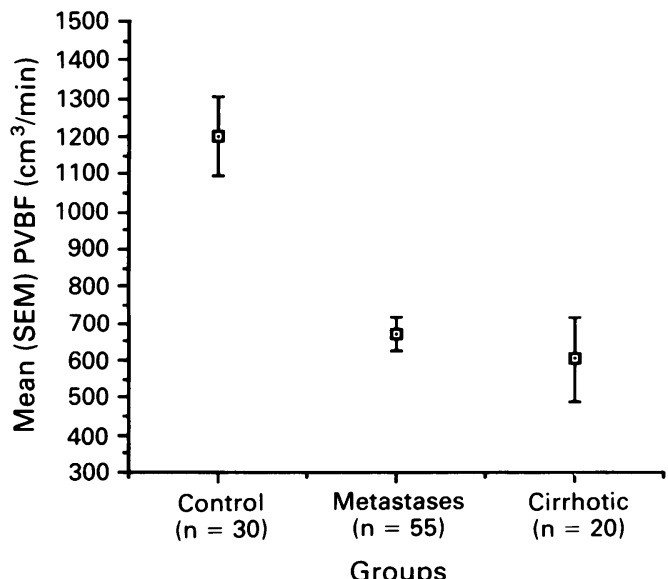

Figure 2: Portal venous blood flow (PVBF) in control subjects and in patients with hepatic metastases and those with cirrhosis. 


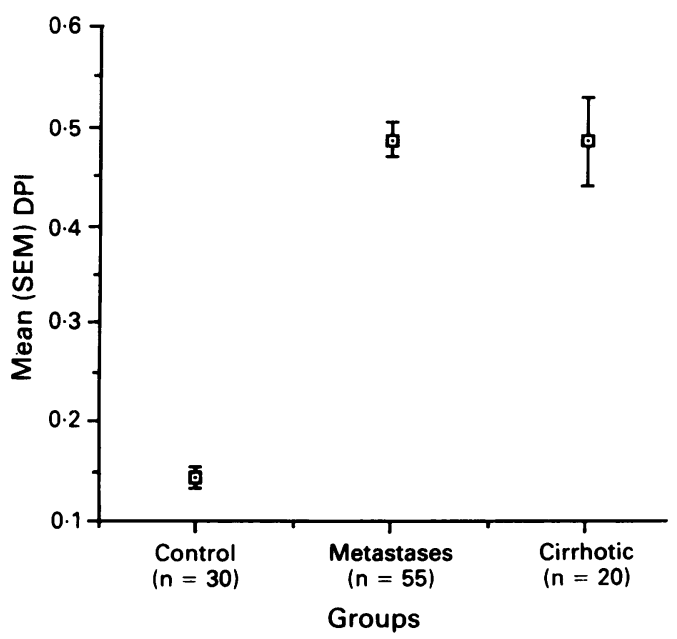

Figure 3: Doppler perfusion index (DPI) in control subjects and in patients with hepatic metastases and those with cirrhosis.

No significant difference was demonstrated in the HCIs among the three groups.

The hepatic arterial resistive indices of the metastatic and cirrhotic groups were significantly reduced compared with those in controls $(\mathrm{p}<0.0001$ and $\mathrm{p}<0.0001)$, reflecting the increase in the hepatic arterial blood flow in the former groups. The ratio of the cross sectional area of the hepatic artery to that of the portal vein was significantly higher in the metastatic group than in the control and the cirrhotic groups $(\mathrm{p}<0.0001, \mathrm{p}<0.0001)$.

\section{Discussion}

Recent technical advances in Doppler sonography have led to the popular use of duplex and colour Doppler scanners to evaluate the haemodynamics of abdominal organs. While there had been numerous studies investigating hepatic cirrhosis using this imaging technique, until now most have been directed towards the haemodynamics of the portal vein, its branches and collaterals, without evaluation of the hepatic arterial component..$^{6-8}$

Although there are still discussions about the accuracy and reproducibility of quantitative

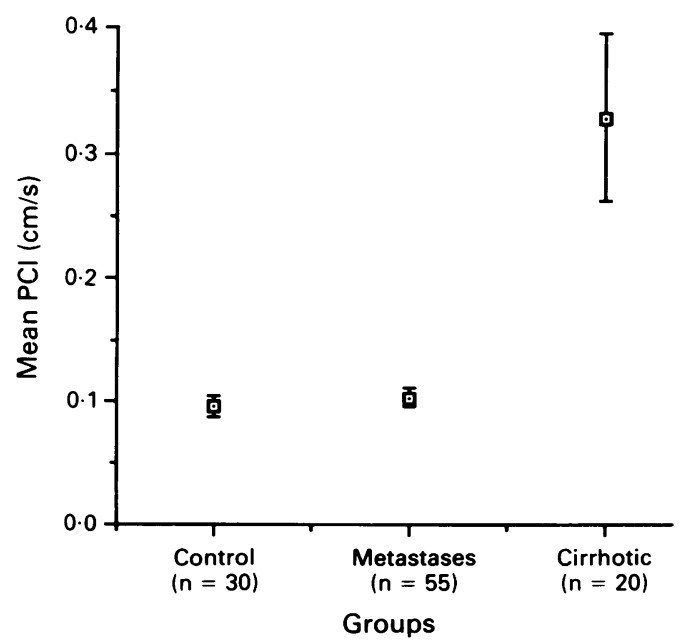

Figure 4: Portal congestive indices (PCI) in control subjects and in patients with hepatic metastases and those with cirrhosis. measurements, ${ }^{9-12}$ the value of qualitative findings is widely accepted. ${ }^{13}$ In a previous study, we carried out in vitro validation tests using a flow circuit model of tissue equivalent material in order to calibrate the DCDS scanner against an electromagnetic flow meter. These had shown that only a single multiplicative correction factor was required to obtain accurate flow measurements in absolute units in vessels of a wide range of diameters for both pulsatile and continuous flow. ${ }^{1+}$ Tests performed on control subjects and patients with liver metastases confirmed that DCDS measurement are highly reproducible..$^{15}$ The main sources of random error in the method arise from uncertainty as to whether the flow in the vessel is parallel to the corrected Doppler cursor and from errors in determining the cross sectional area of the vessel. Systematic errors arise mainly from non-uniform isonation of the vessel and errors in the processing of the Doppler ultrasound signal. Taking all sources of error into account, we estimate that the flow values are accurate to within $25 \% .{ }^{14}$

In a previous study using DCDS, we measured the blood flow changes in the portal vein and the hepatic artery in patients with colorectal liver metastases. ${ }^{5}$ We showed that the measurement of DPI may be useful in the diagnosis of small hepatic tumours and preliminary data suggest that it may even detect occult hepatic metastases. We suspected from results of previous studies using dynamic scintigraphy and measuring HPI, that DPI might be affected similarly in hepatic cirrhosis. Dynamic scintigraphy is limited in principle, however, as it is an indirect method of flow measurement dependent on the hepatic function itself. Any functional reticulo-endothelial impairment would cause inaccuracies in true perfusion changes. On the other hand, DCDS can offer quantitative and qualitative analysis of hepatic haemodynamic changes. The possibility of increasing the specificity of the technique was therefore explored.

Our suspicion was confirmed - all but three patients with cirrhosis had abnormally raised DPI values. While the hepatic perfusion changes in cirrhosis seem to be similar to those in liver metastases (that is, increased hepatic arterial and reduced portal venous blood flows), the cross sectional area ratio of the hepatic artery to portal vein and the portal congestive index were significantly different -51 of $55(93 \%)$ patients with liver metastases had PCI values lower than those of cirrhotics.

This is not altogether surprising since the mechanism underlying the perfusion changes in liver metastases is different from that in cirrhosis. In the case of the former, there is now experimental evidence to suggest that the haemodynamic changes are the result of a circulating vasoactive agent causing an increase in the splanchnic vascular resistance thereby reducing the portal venous blood flow. ${ }^{16} 17$ This would explain the changes associated with small metastases. With larger tumours, however, mechanical obstruction and arterio-venous shunting are probably contributary. The increase in hepatic arterial blood flow in patients with a tumour may be the result of an inherent 
hepatic homeostatic mechanism for the maintainance of total liver blood flow (TLBF) which has been observed in non-tumour bearing animal models and in man. ${ }^{18}$ Indeed no significant difference was noted in the TLBF of controls and that of patients with liver metastases.

In contrast, we know that in cirrhosis the obstruction to portal venous blood flow is at the level of the hepatic sinusoids. There is also a forward flow component resulting from the associated hyperdynamic circulation with increase in cardiac output and decrease splanchnic arteriolar resistance probably caused by some circulating endogenous vasoactive agents. The back pressure in the portal venous system results in the dilatation of the vein and reduction in the time averaged velocity. This is in keeping with the marked increase in the portal congestive index of cirrhotics compared with those of controls as well as the metastatic group. The severity of these changes is dependent on the extent of intra and extrahepatic portosystemic shunting. The significant reduction in total liver blood flow in patients with cirrhotics is in keeping with an intrahepatic obstruction of portal venous blood flow into the liver. The reciprocal increase in the hepatic arterial blood flow may not have been sufficient to maintain total liver blood flow because of excessive intra or extrahepatic portosystemic shunting, or both.

The hepatic arterial resistive index (HARI) is a measure of the arterial vascular impedance. ${ }^{10}$ The reduction of the HARI reflected the increase in the hepatic arterial blood flow in patients with hepatic metastases and those with cirrhosis compared with controls. There was, however, too much overlap between HARI values of the three groups for there to be any diagnostic value in this ratio but it may be useful in longitudinal studies assessing response to therapy.

These data suggest DCDS may differentiate between the hepatic haemodynamic changes associated with cirrhosis and those caused by liver metastases. The measurement of the portal congestive index may increase the diagnostic power of the technique, but further studies are required to confirm these results.

This study was supported by The Scottish Hospital Endowment Research Trust. The authors thank Mrs M Rae and Mr B Jaffray.

1 Parkin A, Robinson PJ, Baxter P, Leveson SH, Wiggins PA Gilles GR. Liver perfusion scintigraphy: method, normal range and laparotomy correlation in 100 patients. Nucl Med Commun 1983; 4: 395

2 Leveson SH, Wiggins PA, Giles GR, Parkin A, Robinson PJ. Deranged liver blood flow patterns in the detection of liver metastases. Br F Surg 1985; 72: 128-30.

3 Laird EE, Williams D, Williams ED. Can the hepatic perfusion index improve routine diagnosis of liver disease? Nucl Med Commun 1987; 8: 959 .

4 Leen E, Goldberg JA, Robertson J, Sutherland GR, McArdle CS. The use of duplex sonography in the detection of
colorectal hepatic metastases. Br $\mathcal{F}$ Cancer 1991; 63: 323-5.

5 Leen E, Goldberg JA, Robertson J, Sutherland GR, Heming way DM, Cooke TG et al. Detection of hepatic metastases using duplex/colour Doppler sonography. Ann Surg 1991; 214 (5): 599-604

6 Ohnishi K, Saito M, Sato S et al. Portal venous haemodynamic in chronic liver disease: effects of postural change and exercise. Radiology 1985; 155: 757-61.

7 Okazaki K, Miyazaki M, Ohnishi S, Ito K. Effect of food intake and various extrinsic hormones on portal blood flow in patients with liver cirrhosis demonstrated by pulsed in patients with liver cirrhosis demonstrated by pulsed
Doppler with the Octoson. Scand f Gastroenterol 1986; 21 : Doppler

8 Gaiani S, Bolondi L, Li Basi S, Santi V, Zironi G, Barbara L. Effect of meal on portal haemodynamics in healthy humans and in patients with chronic liver disease. Hepatology 1989 9: $815-19$

9 Ohnishi K, Saito M, Koen H, Nakayama T, Nomura F, Okuda K. Pulsed Doppler flow as a criterion of portal venous velocity: comparison with cineangiographic measurement. Radiology 1985; 154: 495-8.

10 Gill RW. Measurement of blood flow by ultrasound: accuracy and sources of error. Ultrasound Med Biol 1985; 11: 625-41.

11 Moriyasu F, Ban N, Nishida O, et al. Clinical application of an ultrasonic duplex system in the quantitative measurement of portal blood flow. $\mathscr{C} C U$ 1986; 14: 579-88.

12 Burns PN, Taylor KJW, Blei AT. Doppler flowmetry in portal hypertension. Gastroenterology 1987; 92: 824-6.

13 Barbara L. The value of Doppler US in the study of hepatic haemodynamics. F Hepatol 1990; 10: 353-5.

14 Robertson J, Leen E, Goldberg JA, et al. Flow measurement using duplex Doppler ultrasound: haemodynamic changes in patients with colorectal liver metastases. Clin Physics and Physiological measurements. 1992; 13 (2): 709-18.

15 Leen E, Goldberg JA, Robertson J, et al. Duplex Dopple ultrasound: a novel technique to detect colorectal liver metastases. $\mathcal{F}$ Clin Ultrasound 1993 (in press)

16 Nott DM, Grime SJ, Yates J, Day DW, Baxter JN, Jenkins $\mathrm{SA}$, et al. Changes in the hepatic perfusion index during the development of experimental liver tumours. Brf Surg 1989; 76: 259.

17 Carter R, Anderson JH, Cooke TG, et al. Is the change in the hepatic perfusion index in the presence of hepatic metastases hepatic perfusion index in the presence of hepatic
due to a humoral agent? $\mathrm{Br} \mathcal{F}$ Cancer $1992 ; 65: 58$.

18 Mathie RT, Lam PHM, Harper M, Harper M, Blumgart LH The hepatic arterial blood flow response to portal vein occlusion in the dog: the effect of hepatic denervation. Pflugers Arch 1980; 386: 77. 